\title{
TEMPORAL AVERAGING FOR QUANTIFICATION OF LUMEN DIMENSIONS IN INTRAVASCULAR ULTRASOUND IMAGES
}

\author{
Wenguang Li ${ }^{\dagger \S}$ Elma J. Gussenhoven, ${ }^{\dagger \S}$ Yin Zhong $^{\dagger}{ }^{\dagger}$ Salem H. K. The, ${ }^{\dagger \S}$ \\ Herman Pieterman, ${ }^{\ddagger}$ Hero van URK* and KlaAs BoM ${ }^{\dagger \S}$ \\ Departments of ${ }^{\dagger}$ Thoraxcenter, ${ }^{\dagger}$ Radiology and *Vascular Surgery, University Hospital Rotterdam-Dijkzigt, \\ Erasmus University Rotterdam and ${ }^{\S}$ The Interuniversity Cardiology Institute, The Netherlands
}

(Received 4 May 1993; in final form 27 August 1993)

\begin{abstract}
Quantitative analysis of arterial dimensions from high frequency intravascular ultrasound images (30 MHz) may be hampered by strong blood scattering. Replacement of blood by saline is one method to provide a clear view of the arterial lumen; another method is that of temporal averaging of successive ultrasound images. The accuracy of this latter method was tested by comparing the lumen area measurements on the temporal-averaged image, with the data of the same cross-section obtained from the singleframe and saline-filled images. The mean lumen area measured on the temporal-averaged images was similar to that measured on the single-frame images (mean difference: $-0.02 \pm 1.16 \mathrm{~mm}^{2} ; p=$ ns). The mean lumen area of the saline-filled images was $8 \%$ larger than the values obtained from the temporalaveraged and single-frame images (mean difference: $-1.14 \pm 0.85 \mathrm{~mm}^{2}, p<0.05$ ), probably due to the difference in sound velocity between saline and blood. Intraobserver variation in the averaging method were 2.4 times smaller than the measurements of the single-frame images and close to the data obtained by saline injection (variation coefficient: single-frame: $8.8 \%$; temporal-averaged: $3.6 \%$; saline-filled: $2.9 \%$ ). It is concluded that analysis from temporal-averaged images is more efficient, enabling accurate and reproducible measurement of the luminal dimensions from images containing blood scattering echoes. This technique is suitable to replace the laborious saline injection method and facilitates off-line quantitative analysis.
\end{abstract}

Key Words: Quantitative analysis, Lumen dimensions, Blood interference, Temporal averaging.

\section{INTRODUCTION}

Intravascular ultrasonic imaging provides a direct and dynamic view of arterial vessels in the assessment of atherosclerotic diseases. The use of high frequency ultrasound is essential to achieve high resolution imaging of the arterial structures (Bom et al. 1989; Gussenhoven et al. 1989; Potkin et al. 1990). At high frequency, the echogenicity of blood increases rapidly due to the frequency dependence of blood scatterers, reaching a level similar to that of the arterial tissues (Linker et al. 1991; Lockwood et al. 1991; Shung et al. 1988). The presence of strong blood scattering may mask the leading echoes from the arterial tissues, causing difficulty in discrimination of the luminal boundary. To solve this problem, saline may be injected during clinical examination to replace echogenic blood. Injection of saline is, however, time-consuming and generally

Address correspondence to: E. J. Gussenhoven, Thoraxcenter (Ee 2312), Erasmus University Rotterdam, P.O. Box 1738, 3000 DR Rotterdam, The Netherlands. considered as disadvantageous (Gussenhoven et al. 1991; The et al. 1992a; van Urk et al. 1991). Therefore, we developed a temporal averaging technique to facilitate quantitative off-line analysis of the free lumen area of those ultrasonic images containing blood scattering echoes inside the arterial lumen.

This study was designed to investigate the utility of the temporal averaging method for quantification of the luminal area by comparing measurements on the temporal-averaged image with data from the singleframe image and saline-filled image of the same arterial site.

\section{METHODS}

\section{Intravascular ultrasound}

The intravascular ultrasound imaging system used (Du-MED, Rotterdam, The Netherlands) has a $30 \mathrm{MHz}$ single-element transducer mounted on the tip of a $5 \mathrm{~F}$ catheter. Cross-sectional images were scanned at a rate of 16 frames per second by fast rotation of the trans- 
ducer with a drive shaft $(1000 \mathrm{rpm})$. The system has a bandwidth of $20-40 \mathrm{MHz}$. Axial resolution of the system is $75 \mu \mathrm{m}$ and lateral resolution is better than $225 \mu \mathrm{m}$ at a depth of $2-4 \mathrm{~mm}$. The ultrasonic scan was stored in a $512 \times 512 \times 8$ bits digital memory and displayed with a standard video output via a realtime digital scan converter. Physiological signals, such as electrocardiogram and blood pressure, were simultaneously acquired and superimposed on the video output as a time reference for off-line analysis.

\section{Patient study}

The intravascular ultrasound study was approved by the local committee on Human Research and informed consent was obtained from each patient. Intravascular ultrasound data were acquired from $18 \mathrm{pa}$ tients (male/female: $14 / 4$, age: $52-83$ years) who underwent vascular intervention of the superficial femoral artery for disabling claudication. The echo catheter was advanced via a 7F sheath into the superficial femoral artery. A series of cross-sections was recorded during pull-back of the catheter. At each level of interest, the catheter was kept in position for a period of time sufficient for recording (Gussenhoven et al. 1991; van Urk et al. 1991). If necessary, $5 \mathrm{~mL}$ of heparinized saline solution was injected manually through the introducer sheath to flush out the echogenic blood. The ultrasonic images were recorded on a standard VHS videotape for off-line analysis.

\section{Temporal averaging}

Using real-time intravascular ultrasound, it can be seen that the backscatter pattern of flowing blood varies over time, whereas echoes of arterial tissues present a more static pattern. This phenomenon facilitates differentiation of blood scattering from the echoes of the arterial wall on real-time intravascular ultrasound. The difference can be demonstrated by plotting the intensity profile at one angular position for all the images of one cardiac cycle (Fig. 1). Because the blood scattering changes with time, averaging a number of consecutive images over time may smooth out the scattering from blood while preserving the relatively stable structure of the arterial wall, and thus enhance the contrast at the luminal interface on a still-frame image. Averaging is performed using the following formula:

$$
I_{\text {avg }}(x, y)=\left(I_{1}(x, y)+I_{2}(x, y)+\cdots+I_{n}(x, y)\right) / n .
$$

Here, $I$ presents image intensity, $n$ is the number of consecutive frames at the same cardiac cycle and $x, y$ are image coordinates. This processing is able to cancel out the time-varying signals of blood scatterers and produce a homogeneous luminal region in the image.

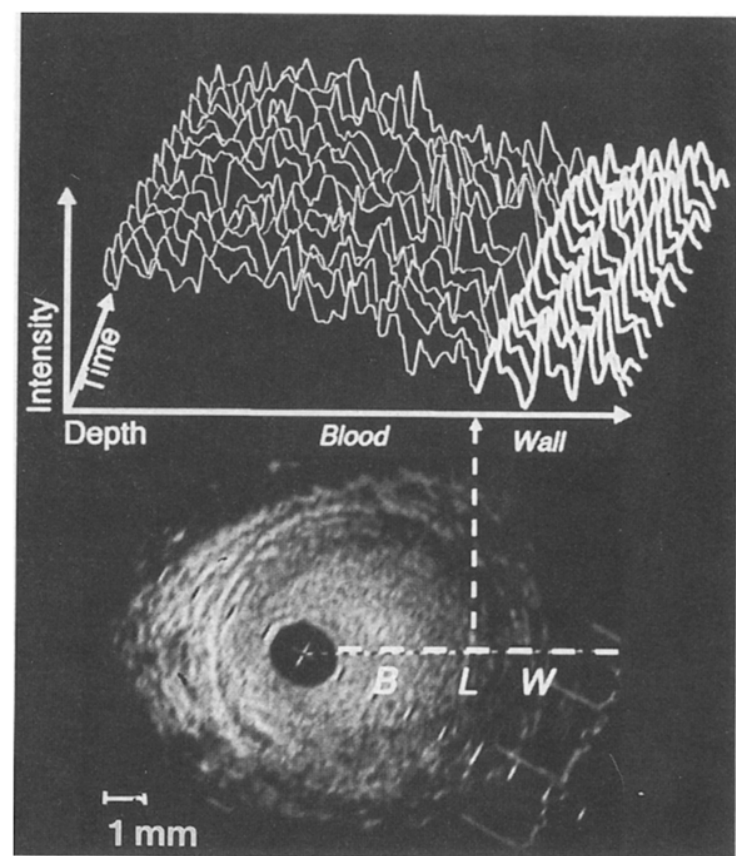

Fig. 1. Plot of intensity profile during one cardiac cycle showing the random changes in backscatter pattern of blood echoes (B; thin line), while the profile of the arterial wall (W) presents a similar pattern during the cardiac cycle (thick line). Shown on the bottom image are the corresponding positions. The dotted horizontal line indicates the angular position at which the plot of the intensity profiles of blood and arterial wall is made. Note that on this intravascular ultrasound cross-section from the superficial femoral artery, the presence of blood hampered visibility of the luminal interface $(\mathrm{L})$.

Of major concern for this algorithm is the possible blurring effect caused by the motion of the wall or the catheter tip. During imaging of peripheral arteries, the relative movement between the catheter and the wall is mainly produced by the wall compliance due to pressure changes. As this wall motion is shown to be minimal in the diastolic period of the cardiac cycle, the blurring effect on the arterial structure can be minimized by selecting the diastolic frames for the averaging procedure (The et al. 1992b).

Theoretically, an averaging process reduces the noise level of a signal by the square root of the number of averaged frames. Figure 2 demonstrates the relationship between the improvement of the signal-to-noise $(\mathrm{S} / \mathrm{N})$ ratio and the number of frames averaged. The signal level is calculated by the mean intensity of the blood scattering; the noise level is defined as the standard deviation of the signal. It has been shown that the $\mathrm{S} / \mathrm{N}$ ratio is improved with the increase of the frame number. After averaging eight frames, there is no significant improvement observed. This is in agreement with our experience that averaging four to eight dia- 


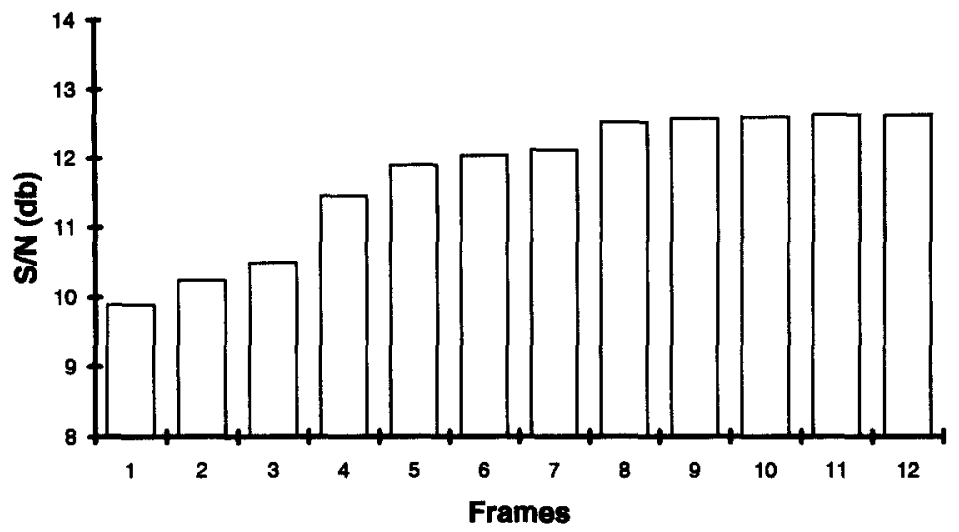

Fig. 2. Values of signal-to-noise $(\mathrm{S} / \mathrm{N})$ ratio as a function of the number of the averaged frames calculated from the data shown in Fig. 1. Note the increase in the $S / N$ ratio from frames $2-8$, and no change in the $S / N$ ratio from frames 8-12.

stolic frames (mean: $N=6$ ) produces satisfactory enhancement of the luminal boundary for the subsequent manual tracing (Fig. 3).

\section{Quantitative measurement}

A computer analysis system based on an IBM compatible PC/AT was used for off-line analysis ( $\mathrm{Li}$ et al. 1991). The system was equipped with a DT 2851 framegrabber to convert video images into $512 \times 512$ $\times 8$ bits digital format. The luminal contour was traced manually with a PC mouse. The absolute measure of the cross-sectional lumen area was calculated by con- verting the pixels into square millimetres with system calibration based on the sound velocity of blood.

Quantitative measurements were made from the intravascular ultrasound images derived from 18 patients, studied prior to or following vascular intervention. At each of 78 arterial sites selected, the free lumen area was measured on: the single-frame, the temporalaveraged and the saline-filled images, respectively (Fig. 4). The single-frame image was selected from the first frame of the image sequence to be averaged. Real-time video images were reviewed to facilitate discrimination of the luminal interface from blood. To test

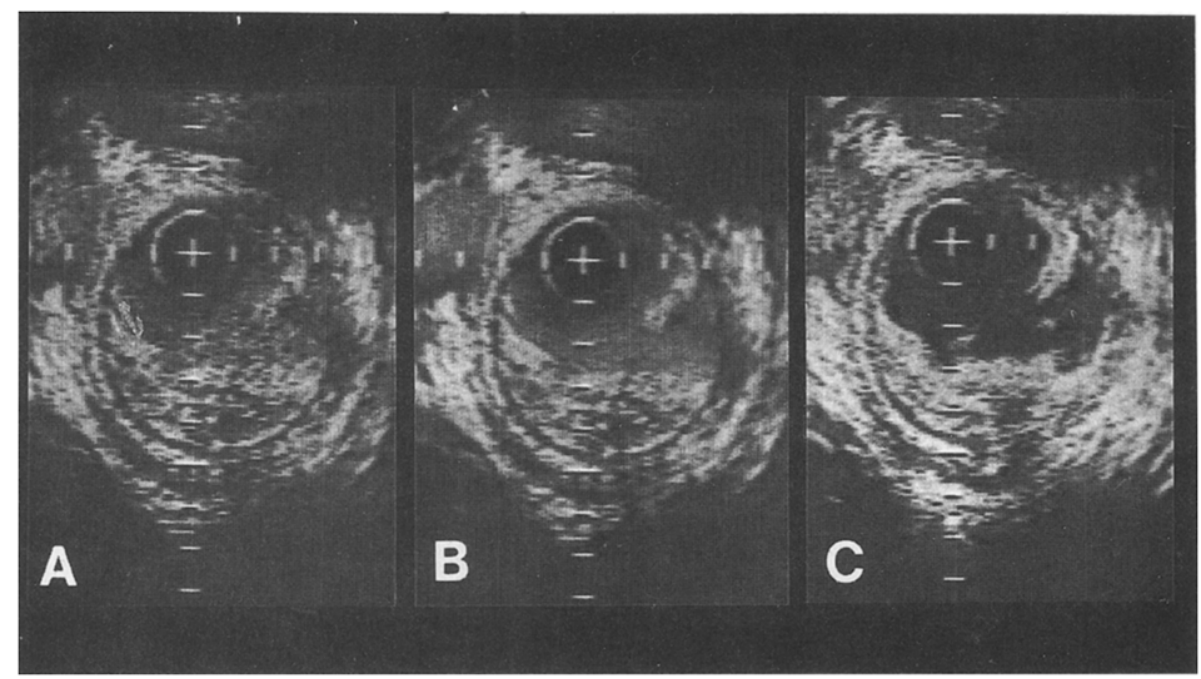

Fig. 3. Three different presentations of an ultrasound cross-section from a superficial femoral artery (scale mark: $1 \mathrm{~mm}$ ). (A) single-frame image containing the blood scattering; (B) temporal-averaged image; and (C) image after saline injection. The visibility of the luminal structure is significantly improved on the temporal-averaged image and the saline-filled image, particularly the edge of the ruptured lesion, which is difficult to recognize on the single-frame image. 


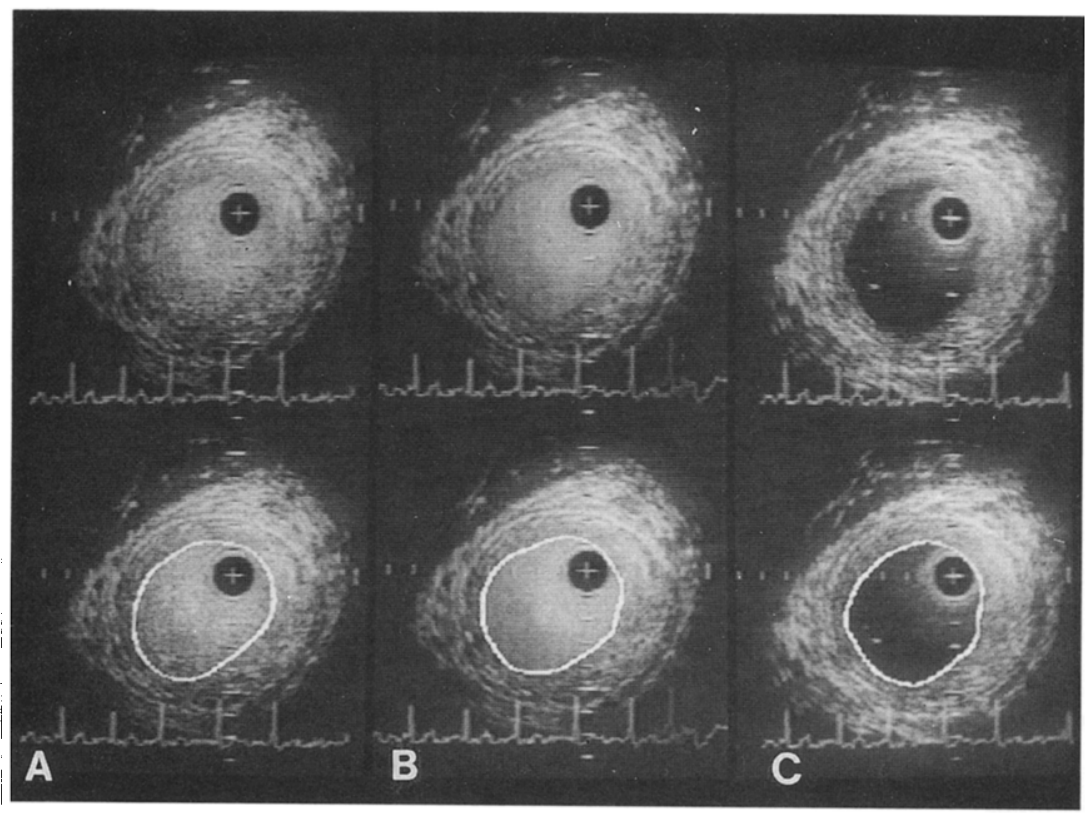

Fig. 4. Contour tracing of the free lumen area on the single-frame image (A), the temporal-averaged image (B) and the image with saline solution (C) (scale mark: $1 \mathrm{~mm}$ ). (A) the luminal contour has to be estimated by reviewing the real-time images; $(\mathrm{B})$ the temporal-averaging method allows direct tracing of the luminal contour; and $(C)$ the use of saline solution enables a clear definition of the free lumen but introduces deviations in measurements due to the differences in sound velocity between blood and saline.

the reproducibility of the three methods, measurements were repeated by the same observer two weeks later.

\section{Data analysis}

The three series of measurements are presented with the mean values and standard deviations (SD). Comparison of two measurements in each cross-section was performed by calculating the mean and SD of the paired differences. The significance of the difference of the mean values was determined by a paired $t$-test. A probability value of $<0.05$ was considered statistically significant.

The degree of intraobserver variation is presented with a coefficient of variation defined as: (SD of the paired differences/mean value of the measurements) $\times 100 \%$.

\section{RESULTS}

The mean lumen areas measured on single-frame, temporal-averaged and saline-filled images were 13.86 $\pm 6.30 \mathrm{~mm}^{2}, 13.88 \pm 6.15 \mathrm{~mm}^{2}$ and $15.00 \pm 6.76 \mathrm{~mm}^{2}$, respectively (Fig. 5). The mean difference between the temporal-averaged and single-frame images was minimal (paired difference: $-0.02 \pm 1.16 \mathrm{~mm}^{2}, p=$ ns). Comparing measurements from the temporal-averaged images with saline-filled images showed the mean value of the latter method to be $8 \%$ larger (paired difference: $-1.14 \pm 0.85 \mathrm{~mm}^{2}, p<0.05$ ).

The coefficient of intraobserver variation was $8.8 \%$ for data from single-frame images, $3.6 \%$ for data from temporal-averaged images and $2.9 \%$ for measurements on saline-filled images (Fig. 6).

\section{DISCUSSION}

Intravascular ultrasound has shown promise as a safe and accurate imaging modality for detection of atherosclerosis as well as for the assessment of the outcome of vascular intervention (Gussenhoven et al. 1991; Nissen et al. 1990; Tobis et al. 1991; van Urk et al. 1991). The limitation encountered using high frequency ultrasound during off-line quantitative anal$y$ sis of the free lumen area is the echogenicity of blood. In contrast, off-line quantitative analysis using the temporal averaging method permits a significant improvement in visualization of the luminal boundary on a still-frame image. Although the averaging procedure does not remove the echoes of blood scatterers, it generates a homogeneous luminal region by smoothing out the time-varying signals.

The results of this study show no differences in the mean measurements between the single-frame images and the data obtained by the averaging method. The mean lumen area measured on the saline-filled 

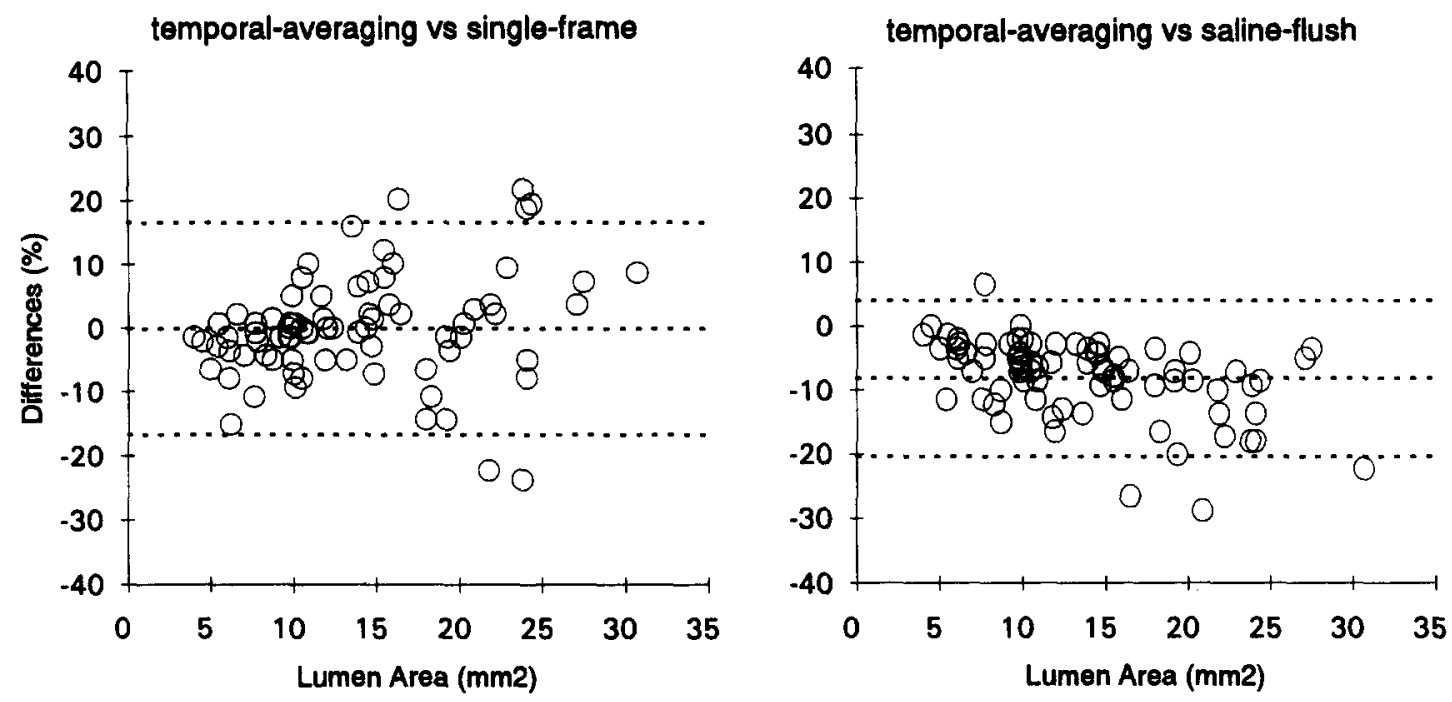

Fig. 5. The differences in the lumen area measurements obtained by the temporal averaging method and the single-frame images (left), and the saline-filled images (right). The $95 \%$ confidence interval is indicated by the upper and lower horizontal lines (mean $\pm 2 \mathrm{SD}$ ).

images was $8 \%$ larger than that of both the singleframe and the averaged images. One reason for this discrepancy may be the difference in ultrasound velocity between blood and saline. Saline solution has a lower ultrasound velocity than blood (saline: $1540 \mathrm{~m} /$ s vs. blood: $1580 \mathrm{~m} / \mathrm{s}$ at $37^{\circ} \mathrm{C}$; a difference of $2.6 \%$ ). Theoretically, a $2.6 \%$ change in sound velocity may produce an approximately $5.2 \%$ difference in area measurement of a circular lumen. In an in vitro setting, Moriuchi and co-workers (1990) found that the mean lumen area in saline increased 6\%, compared with that in blood-filled images. Assuming that most of the intravascular ultrasound imaging systems are calibrated with the sound velocity in blood, the measurement of an image filled with saline solution will result in an overestimation of the vessel dimension. The second factor that may lead to an increase of the luminal area during saline injection is the initial increase in the internal vascular pressure due to the injection.

In this study, the data obtained from a well-defined lumen during saline injection were used as a reference to test the reproducibility of the temporal averaging method. The measurement reproducibility using the averaging method was improved 2.4 times compared to that of single-frame images, and was similar to the results obtained from the saline-filled images
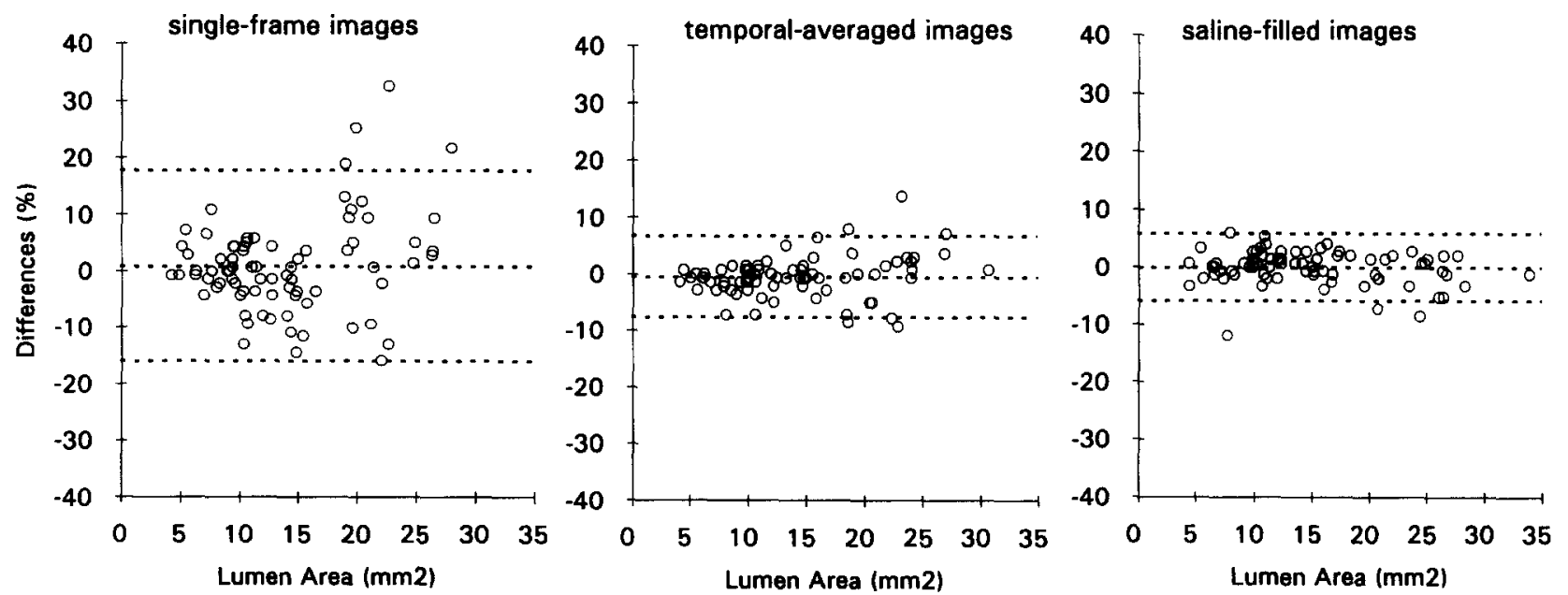

Fig. 6. Intraobserver variations for free lumen area $\left(\mathrm{mm}^{2}\right)$ using three different methods. The $95 \%$ confidence interval is indicated by the upper and lower horizontal lines (mean \pm 2 SD). 
(variation coefficient: single-frame: $8.8 \%$, temporalaveraged image: $3.6 \%$, saline-filled image: $2.9 \%$ ). The higher intraobserver variation for measurements of single-frame images is due to the lack of a luminal contrast with the presence of blood scattering. One has to estimate the location of the contour by memorizing information from real-time video images; this procedure is time-consuming as the operator needs to review the video tape frequently.

Because the averaging procedure improves the signal-to-noise ratio, one may expect that measuring the averaged image would have a similar effect as the averaging of several individual measurements from the single-frame images. This means the variation coefficient will be reduced by the square root of the number of the measurements. Our results indicate that the improvement in measurement reproducibility by the averaging method is comparable to that of approximately six measurements of single-frame images. The temporal averaging method, however, is more efficient. The technique is easy to implement in an off-line system and requires a few seconds for the selection of frames to be processed.

Although the visibility of blood is considered as disadvantageous for off-line analysis, it should be emphasized that during real-time imaging, the arterial lumen can be differentiated by the characteristic changes in the blood scattering pattern. Particularly in the case of complex arterial dissections following balloon angioplasty, the visibility of blood may be helpful in assessing the anatomic substrate. The use of saline injection is mainly to facilitate off-line quantitative analysis.

\section{CONCLUSION}

This study has demonstrated the feasibility of using the temporal averaging method to enhance the luminal boundary of intravascular ultrasound images in the presence of strong interference of blood scatterers. The technique is able to reduce observer variation in single-frame images and produce a comparable performance as the saline flush method. Hence, the temporal averaging method, which provides an accurate and reproducible measure of the luminal dimensions in the presence of blood, may obviate the need of laborious saline injections during the catheterization procedure.

Acknowledgements - The authors are indebted to H. Rijsterborgh for his comments. This project was supported by a grant from the Interuniversity Cardiology Institute of the Netherlands and the Netherland Heart Foundation.

\section{REFERENCES}

Bom, N.; ten Hoff, H.; Lancée, C. T.; Gussenhoven, E. J.; Bosch, J. G. Early and recent intraluminal ultrasound devices. Int. J. Cardiac Imag. 4:79-88; 1989.

Gussenhoven, E. J.; Essed, C. E.; Lancée, C. T.; Mastik, F.; Frietman, P. Arterial wall characteristics determined by intravascular ultrasound imaging: An in-vitro study. J. Am. Coll. Cardiol. 14:947-952; 1989.

Gussenhoven, E. J.; Frietman, P. A. V.; The, S. H. K.; van Suylen, R. J.; van Egmond, F. C. Assessment of medial thinning in atherosclerosis by intravascular ultrasound. Am. J. Cardiol. 68:1625-1632; 1991

Li, W.; Gussenhoven, W. J.; Zhong, Y.; The, S. H. K.; Di Mario, C. Validation of quantitative analysis of intravascular ultrasound images. Int. J. Cardiac Imag. 6:247-253; 1991.

Linker, D. T.; Kleven, A.; Grønningsæather, А.; Yock, P. G.; Angelsen, A. J. Bj. Tissue characterization with intra-arterial ultrasound: Special promise and problems. Int. J. Cardiac Imag. 6:255-263; 1991.

Lockwood, G. R.; Ryan, L. K.; Hunt, J. W.; Foster, F. S. Measurement of the ultrasonic properties of vascular tissues and blood from 35-65 MHz. Ultrasound Med. Biol. 1:653-666; 1991.

Moriuchi, M.; Tobis, J. M.; Mahon, D.; Gessert, J.; Griffith, J. The reproducibility of intravascular ultrasound imaging in-vitro. J. Am. Soc. Echocardiogr. 3:444-450; 1990.

Nissen, S. E.; Grines, C. L.; Gurley, J. C. Application of a new phased-array ultrasound imaging catheter in the assessment of vascular dimensions. In-vivo comparison to cineangiography. Circulation 81:660; 1990.

Potkin, B. N.; Bartorelli, A. L.; Gessert, J. M.; Neville, R. F.; Almagor, Y. Coronary artery imaging with intravascular ultrasound. Circulation 81:1575-1585; 1990.

Shung, K. K.; Yuan, Y. W. Ultrasonic backscatter from flowing whole blood. II: Dependence on frequency and fibrinogen concentration. J. Acoust. Soc. Am. 84:1195-1200; 1988.

The, S. H. K.; Gussenhoven, E. J.; Zhong, Y.; Li, W.; van Egmond, $F$. The effect of balloon angioplasty on the femoral artery evaluated with intravascular ultrasound imaging. Circulation 86:483493; 1992a.

The, S. H. K.; Gussenhoven, E. J.; Li, W.; de Feyter, P.; Serruys, P. W. Intravascular ultrasonic assessment of lumen geometry and distensibility of the angiographically normal artery: A correlation with quantitative angiography. Echocardiography 9:133-139; $1992 b$.

Tobis, J. M.; Mallery, J.; Mahon, D.; Lehmann, K.; Zaleski, P. Intravascular ultrasound imaging of human coronary arteries in vivo. Analysis of tissue characterizations with comparison to in vitro histological specimens. Circulation 83:913-926; 1991.

van Urk, H.; Gussenhoven, E. J.; Gerritsen, G. P.; The, S. H. K. van Egmond, F. Assessment of arterial disease reconstructions by intravascular ultrasound. Int. J. Cardiac Imag. 6:157-164; 1991. 